Revue d'histoire de l'Amérique française

REVUE D.HISTOIRE DE L'AMÉRIQUE FRANÇAISE

\title{
Famille, parenté et colonisation en Nouvelle-France
}

\section{Yves Beauregard, Serge Goudreau, Andrée Héroux, Michèle Jean, Rénald Lessard, Johanne Noel, Lucie Paquet et Alain Laberge}

Volume 39, numéro 3, hiver 1986

URI : https://id.erudit.org/iderudit/304373ar

DOI : https://doi.org/10.7202/304373ar

Aller au sommaire du numéro

\section{Éditeur(s)}

Institut d'histoire de l'Amérique française

ISSN

0035-2357 (imprimé)

1492-1383 (numérique)

Découvrir la revue

Citer cette note

Beauregard, Y., Goudreau, S., Héroux, A., Jean, M., Lessard, R., Noel, J., Paquet, L. \& Laberge, A. (1986). Famille, parenté et colonisation en Nouvelle-France. Revue d'histoire de l'Amérique française, 39(3), 391-405.

https://doi.org/10.7202/304373ar d'utilisation que vous pouvez consulter en ligne. 
NOTE DE RECHERCHE

\title{
FAMILLE, PARENTÉ ET COLONISATION EN NOUVELLE-FRANCE ${ }^{1}$
}

\author{
YVES BEAUREGARD \\ SERGE GOUDREAU \\ ANDRÉE HÉROUX \\ MICHELE JEAN \\ RÉNALD LESSARD \\ JOHANNE NOEL \\ LUCIE PAQUET \\ Université Laval \\ ALAIN LABERGE \\ Université York
}

\section{INTRODUCTION}

La connaissance des sociétés pré-industrielles s'est considérablement élargie depuis plusieurs années grâce aux recherches sur les structures familiales et le rôle de la parenté. Il n'est plus possible aujourd'hui de tenter d'apréhender la réalité sociale passée sans reconnaître à la famille une place et une force hautement déterminante dans les pratiques sociales.

En Nouvelle-France, l'intérêt de ces questions réside surtout dans l'évaluation de la nature et de la fonction de la famille et de la parenté dans un contexte de faible densité démographique. Cette adaptation à un milieu neuf contraste avec l'occupation des terroirs pleins d'Europe où ont été réalisées les premières études sur la famille. L'immigrant français, issu d'une société où la tradition familiale s'exerce depuis des générations, se retrouve dans un environnement physique et social différent. Les ajustements et les évolutions découlant de cette situation représentent autant de pistes de recherche.

Pour sa part, notre groupe de recherche s'est penché sur la formation de nouvelles communautés sous le Régime français. La synthèse de ces recherches nous permet dans un premier temps de tracer un portrait du migrant basé sur une série de données démographiques. En second lieu, nous étudions l'importance des liens familiaux dans le

\footnotetext{
1 Le présent texte a fait l'objet de communications au Congrès de l'Institut d'histoire de l'Amérique française, le 20 octobre 1984 et à celui de la French Colonial Historical Society, le 10 mai 1985. Nous tenons à remercier Jacques Mathieu, professeur au département d'histoire de l'Université Laval et directeur du CELAT. Tout au long de notre démarche, sa disponibilité, son appui et ses avis judicieux ont été d'un précieux secours.
} 
processus d'enracinement de l'arrivant. Cette synthèse constitue un apport nouveau à l'histoire de la colonisation à l'époque de la NouvelleFrance.

\section{1 - LES AIRES D'OBSERVATION}

Le choix des aires d'observation repose uniquement sur les intérêts de recherche des participants, si bien que l'origine informelle et volontaire de notre groupe a produit certains déséquilibres. Par exemple, on ne retrouve qu'une seule seigneurie du gouvernement de Montréal (Chambly) et aucune du gouvernement de Trois-Rivières. De même, les seigneuries dont l'observation débute dans le dernier quart du $17 \mathrm{e}$ siècle sont les plus nombreuses dans notre échantillon.

TABLEAU 1

Les aires d'observation ${ }^{2}$

\begin{tabular}{|llc|}
\hline Seigneuries & \multicolumn{1}{c|}{ Appellation retenue } & Dates d'observation \\
\hline Beaumont & Beaumont & $1672-1709$ \\
Chambly & Chambly & $1668-1694$ \\
Deschambault/Lachevrotière & Deschambault & $1674-1725$ \\
Les Éboulements & Les Éboulements & $1709-1769$ \\
Côte de Gaspé & Gaspé & $1750-1760$ \\
Lotbinière & Lotbinière & $1672-1709$ \\
Aulnaies/Pocatière/ & & \\
Rivière-Ouelle & Rivière-Ouelle & $1672-1704$ \\
Fleury & St-Joseph & $1737-1747$ \\
\hline
\end{tabular}

Ces seigneuries évoquent des contextes de peuplement variés tant par le milieu, le temps, les types, les conditions et les rythmes d'établissement. En somme, elles reflètent les étapes et les différentes formes de pénétration du territoire laurentien.

Deschambault et les seigneuries de la rive sud (Lotbinière, Beaumont et la région de Rivière-Ouelle) reçoivent leurs premiers arrivants dans les dernières décennies du 17e siècle alors qu'elles absorbent les surplus de population que ne peuvent retenir les zones de colonisation plus anciennes. Dans certains cas bien précis, comme à Beaumont et à Rivière-Ouelle, la présence du seigneur sur les lieux, dès le début, garantit une certaine avance dans le peuplement. Mais dans l'ensemble, on sent bien la cadence irrégulière des arrivées comme en font foi les

2 Voir la figure 1. 


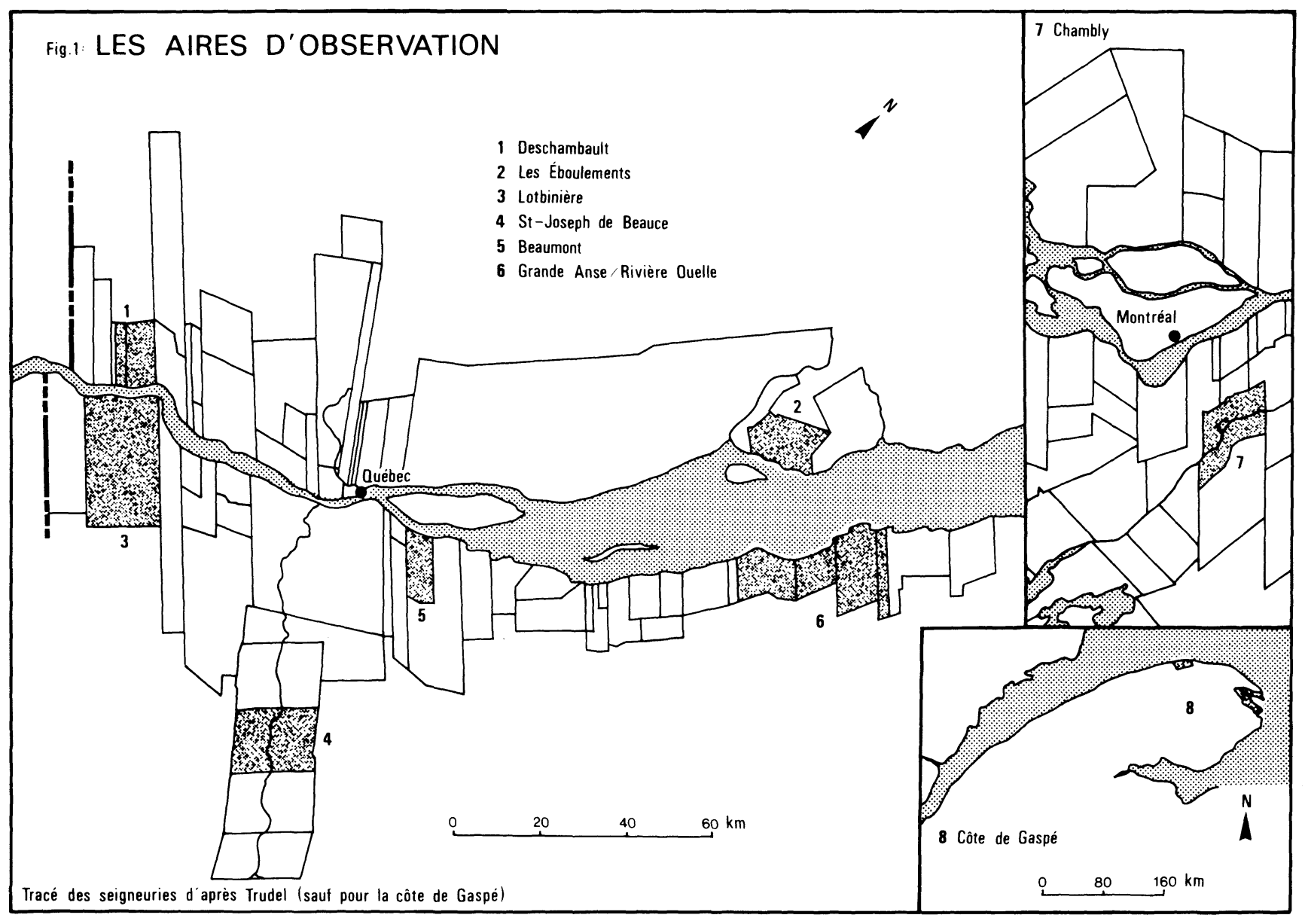


périodes d'observation d'au moins trente ans nécessaires à la composition de corpus assez larges.

A la même époque, mais pour des raisons qui tiennent davantage à la sécurité de la colonie, la seigneurie de Chambly est concédée et les autorités tentent d'y fixer des soldats licenciés. Dans cette communauté frontalière, les arrivées comme les départs se font en vagues successives selon les périodes de paix ou de guerre. Le type particulier des colons et les conditions précaires de leur établissement entraînent l'instabilité du peuplement de Chambly pendant tout le $17 \mathrm{e}$ siècle.

$\mathrm{Au} 18 \mathrm{e}$ siècle, dans une conjoncture de croissance démographique rapide, l'avancée se poursuit et touche des zones excentriques comme la Beauce et Charlevoix. Dans la seigneurie de Fleury (Saint-Josephde-Beauce), le seigneur Joseph Fleury de la Gorgendière voit promptement à la mise en place d'infrastructures favorisant l'implantation des censitaires. Ceux-ci viennent nombreux et en l'espace d'une dizaine d'années, la population de la seigneurie de Fleury atteint un niveau fort respectable. Il en va tout autrement aux Éboulements alors que les fils et les filles du seigneur Pierre Tremblay monopolisent l'espace seigneurial dès le départ, instituant du même coup un type de peuplement très fermé à l'étranger. Les migrants y seront donc en petit nombre.

Enfin, les seigneuries de la côte de Gaspé représentent une région où l'exploitation d'une ressource particulière, la pêche, détermine le caractère saisonnier des migrations. Dans ce sens, les communautés de pêcheurs de Gaspésie sont évidemment bien différentes de celles des autres aires d'observation où prédomine l'agriculture. Cependant, dans le cadre de la présente étude, le cas de la côte de Gaspé est une occasion de considérer l'influence des liens familiaux sur un type de migration qui semble attirer de plus en plus de gens à la fin du Régime français ${ }^{3}$.

Le nombre et la diversité des aires d'observation nous ont incités à élaborer une méthodologie rigoureuse qu'il convient d'exposer brièvement afin de bien comprendre l'usage et la signification de plusieurs termes employés par la suite.

\section{2 - LE CADRE MÉTHODOLOGIQUE}

Il était impératif au départ de relever le plus exactement possible le nombre de personnes ayant migré dans l'une ou l'autre des aires d'observation retenues. A cet égard, les registres d'état civil et les greffes de notaires ont constitué les principaux instruments de repérage. Nous avons complété au besoin par d'autres sources comme le recensement

\footnotetext{
${ }^{3}$ Le corpus utilisé pour Gaspé n'inclut que les individus qui s'engagent devant un notaire du gouvernement de Québec. Les pêcheurs engagés en Europe ne sont pas retenus aux fins de l'étude.
} 
de 1681, la carte de Gédéon de Catalogne, des procès-verbaux d'arpentage et des aveux et dénombrements.

Ces documents nous ont servi à identifier les arrivants à partir des évidences d'établissement. Si des mentions fréquentes dans les registres d'état civil d'une paroisse ou l'acquisition d'une terre dans le même lieu constituaient de bons indices, elles devaient dans la mesure du possible être corroborées par une mention formelle de résidence. Tous les détenteurs de censives n'ont donc pas été considérés comme arrivants mais uniquement ceux qui, pour reprendre l'expression du temps, ont tenu feu et lieu.

On définit comme arrivant chacun des conjoints d'un couple marié, les veufs et les veuves, tout en excluant leurs enfants, même ceux en âge de s'établir. Les célibataires venus sans ou après leurs parents et les conjoints que des résidents iront chercher en dehors de l'aire d'observation sont également considérés comme des arrivants. Au total, notre corpus s'est composé de 608 arrivants.

Chacun des arrivants a fait l'objet d'une fiche signalétique contenant d'abord des renseignements permettant de dresser son portrait, soit: le nom, la date d'arrivée, le sexe, l'âge, le statut matrimonial, l'ancienneté du mariage et le nombre d'enfants à l'arrivée, le lieu de naissance et la provenance.

Viennent ensuite les données relatives aux liens familiaux. Nous avons privilégié trois situations de parenté. La première, la parenté à l'arrivée, s'applique à un groupe d'arrivants parents entre eux et venus investir un espace ensemble. Elle concerne également un arrivant qui va rejoindre un ou des parents déjà sur place. La deuxième situation, la parenté attirée, est simplement le pendant de ce dernier cas et se rapporte $\mathrm{au}(\mathrm{x})$ parent(s) en question. Troisièmement, il y a la parenté créée qui consiste en la formation de liens de parenté entre les résidants et qu'on retrouve exclusivement par le biais des mariages et des remariages.

Enfin, après un suivi de chacun des arrivants, nous avons pu déterminer la permanence ou non de leur établissement. L'acte de sépulture est évidemment la meilleure preuve d'une installation permanente dans la communauté. Cela implique toutefois que des arrivants qui meurent peu de temps après leur arrivée se trouvent classés comme étant permanents tandis que d'autres arrivants qui repartent même plusieurs années après leur venue se rangent dans la catégorie des migrants temporaires.

Un tel cadre méthodologique n'élimine cependant pas les difficultés rattachées à l'état de la documentation utilisée. Des registres paroissiaux manquants et des concessions de terre ou des transactions sous seing privé disparues peuvent cacher des arrivants, nécessairement tem- 
poraires dans ce cas, qui n'auraient pas laissé d'autres traces de leur passage. Malgré cela, nous avons la conviction que notre corpus rejoint la très grande majorité des arrivants.

\section{3 - LE PORTRAIT DE L'ARRIVANT}

Diverses stratégies de peuplement caractérisent l'appropriation du territoire laurentien. Les paroisses agricoles situées à proximité des anciennes zones de colonisation se distinguent nettement de Chambly et de Gaspé. En conséquence, le portrait du migrant sera sensiblement différent à l'intérieur des communautés rurales de ce qu'il est dans des lieux marqués par le caractère saisonnier ou temporaire de l'établissement.

Tracer un profil général de l'arrivant constitue une étape essentielle. A cette fin, nous avons retenu les caractéristiques suivantes: lieu de naissance, âge à l'arrivée et statut matrimonial.

Le tableau 2 nous permet de constater que plus de la moitié des arrivants sont des Canadiens de naissance.

TABLEAU 2

Lieu de naissance de l'arrivant, par aire d'observation

\begin{tabular}{|c|c|c|c|c|c|c|c|c|}
\hline \multirow{3}{*}{$\begin{array}{l}\text { Aire } \\
\text { d'observation }\end{array}$} & \multicolumn{8}{|c|}{ Lieu de naissance } \\
\hline & \multicolumn{2}{|c|}{$\begin{array}{c}\text { Extérieur } \\
\text { colonie }\end{array}$} & \multicolumn{2}{|c|}{ Canada } & \multicolumn{2}{|c|}{ Indéterminé } & \multicolumn{2}{|c|}{ Ensemble } \\
\hline & n.a. & $\%$ & n.a. & $\%$ & n.a. & $\%$ & n.a. & $\%$ \\
\hline Beaumont & 50 & 41 & 65 & 53 & 8 & 6 & 123 & 100 \\
\hline Chambly & 51 & 69 & 5 & 7 & 18 & 24 & 74 & 100 \\
\hline Deschambault & 21 & 22 & 71 & 73 & 5 & 5 & 97 & 100 \\
\hline Les Éboulements & 1 & 3 & 31 & 97 & 0 & 0 & 32 & 100 \\
\hline Gaspé & 29 & 58 & 21 & 42 & 0 & 0 & 50 & 100 \\
\hline Lotbinière & 25 & 55 & 16 & 36 & 4 & 9 & 45 & 100 \\
\hline Rivière-Ouelle & 43 & 41 & 54 & 52 & 7 & 7 & 104 & 100 \\
\hline St-Joseph Beauce & 17 & 20 & 54 & 65 & 12 & 15 & 83 & 100 \\
\hline Ensemble & 237 & 39 & 317 & 52 & 54 & 9 & 608 & 100 \\
\hline
\end{tabular}

n.a.: nombre absolu

Les proportions obtenues varient toutefois considérablement d'une communauté à l'autre. En effet, nous observons à Chambly et sur la côte de Gaspé un grand nombre de gens natifs de l'extérieur de la colonie. 
Cette situation s'explique aisément. Le développement initial de Chambly repose sur un fort contingent d'immigrants recrutés dans les différentes compagnies du régiment de Carignan. Quant à la côte de Gaspé, l'engagement collectif de pêcheurs du pays basque et de la Normandie y prend une importance insoupçonnée jusqu'alors.

Nous constatons également que les communautés qui se développent au $17 \mathrm{e}$ siècle comptent un nombre sensible d'immigrants français. Lotbinière, Beaumont et Rivière-Ouelle ont profité de l'immigration française du dernier tiers du 17e siècle. D'autre part, Deschambault, les Eboulements et St-Joseph doivent une grande part de leur développement à la croissance naturelle des anciennes zones de colonisation. En somme, ces communautés réfèrent à des modes de peuplement diversifiés qui n'excluent pas certaines similarités.

Dans chacune de ces communautés, il semble que le phénomène migratoire touche particulièrement les jeunes adultes (tableau 3). Toutefois, un nombre passablement important de personnes plus âgées apparaissent également comme migrants. Par groupes quinquennaux d'âges, la distribution des arrivants est la suivante pour chacune des aires d'observation:

TABLEAU 3

Distribution des arrivants par groupes quinquennaux d'âges et par aire d'observation

\begin{tabular}{|lrrrrrrrr|}
\hline & \multicolumn{10}{c|}{ Groupes d'âges } \\
\hline $\begin{array}{l}\text { Aire } \\
\text { d'observation }\end{array}$ & $\begin{array}{c}15-19 \\
\text { ans }\end{array}$ & $\begin{array}{c}20-24 \\
\text { ans }\end{array}$ & $\begin{array}{c}25-29 \\
\text { ans }\end{array}$ & $\begin{array}{c}30-34 \\
\text { ans }\end{array}$ & $\begin{array}{c}35-39 \\
\text { ans }\end{array}$ & $\begin{array}{c}40+ \\
\text { ans }\end{array}$ & $\begin{array}{c}\text { Ind. } \\
\text { Ensem- } \\
\text { ble }\end{array}$ \\
\hline Beaumont & 10 & 23 & 20 & 17 & 18 & 27 & 8 & 123 \\
Chambly & 6 & 14 & 11 & 9 & 0 & 9 & 25 & 74 \\
Deschambault & 4 & 19 & 24 & 9 & 13 & 20 & 8 & 97 \\
Les Éboulements & 3 & 9 & 12 & 4 & 0 & 2 & 2 & 32 \\
Gaspé & 3 & 8 & 8 & 6 & 4 & 3 & 18 & 50 \\
Lotbinière & 6 & 6 & 3 & 9 & 6 & 13 & 2 & 45 \\
Rivière-Ouelle & 10 & 22 & 21 & 18 & 11 & 16 & 6 & 104 \\
St-Joseph de Beauce & 5 & 17 & 21 & 9 & 4 & 15 & 12 & 83 \\
\hline Ensemble & & & & & & & & \\
$\quad$ nombre absolu & 47 & 118 & 120 & 81 & 56 & 105 & 81 & 608 \\
$\quad$ nombre relatif $(\%)$ & 9 & 22 & 23 & 15 & 11 & 20 & - & 100 \\
\hline
\end{tabular}

Les migrants se répartissent donc dans un éventail très large des tranches d'âges. On y retrouve un bon nombre de jeunes adultes (54\% 
ont moins de 30 ans) mais signalons que $20 \%$ des arrivants ont plus de 40 ans. Il semble donc, en matière d'âge à l'arrivée, que le portrait du migrant soit tout de même assez diversifié. Il sera d'ailleurs révélateur de distinguer les migrants en fonction de leur état matrimonial à l'arrivée (tableau 4).

TABLEAU 4

État matrimonial de l'arrivant, par aire d'observation

\begin{tabular}{|lrrrrrrrr|}
\hline & \multicolumn{8}{c|}{ État matrimonial } \\
\hline Aire & \multicolumn{1}{c}{ Mariés(es) } & \multicolumn{2}{c|}{ Célibataires } & \multicolumn{2}{c|}{ Veufs(ves) } & \multicolumn{2}{c|}{ Ensemble } \\
d'observation & n.a. & $\%$ & n.a. & $\%$ & n.a. & $\%$ & n.a. & $\%$ \\
\hline Beaumont & 94 & 78 & 21 & 18 & 5 & 4 & 120 & 100 \\
Chambly & 16 & 25 & 47 & 73 & 1 & 2 & 64 & 100 \\
Deschambault & 71 & 76 & 19 & 20 & 4 & 4 & 94 & 100 \\
Les Éboulements & 26 & 81 & 6 & 19 & 0 & 0 & 32 & 100 \\
Gaspé & 22 & 44 & 28 & 56 & 0 & 0 & 50 & 100 \\
Lotbinière & 35 & 78 & 10 & 22 & 0 & 0 & 45 & 100 \\
Rivière-Ouelle & 75 & 72 & 25 & 24 & 4 & 4 & 104 & 100 \\
St-Joseph Beauce & 53 & 64 & 30 & 36 & 0 & 0 & 83 & 100 \\
\hline Ensemble & 392 & 66 & 186 & 32 & 14 & 2 & $592 *$ & 100 \\
\hline
\end{tabular}

* 16 indéterminés n.a.: nombre absolu

Le peuplement de la majorité de ces communautés se réalise par la venue de gens mariés. En effet, $66 \%$ des arrivants sont déjà mariés lors de leur installation. Toutefois, Chambly et Gaspé se distinguent nettement des six autres aires d'observation. A Chambly, on retrouve un modèle particulier de peuplement basé sur l'arrivée massive de célibataires qui devront chercher leur conjointe à l'extérieur. A Gaspé, des préoccupations d'ordre économique prédominent car le peuplement est surtout formé d'hommes célibataires qui pour la majorité ne s'établissent que le temps d'une saison de pêche.

Dans les six autres communautés, il se révèle une constante pour le moins intéressante. Le couple joue un rôle de premier plan dans le mouvement colonisateur. En effet, $74 \%$ de ceux-ci sont déjà mariés à leur arrivée. Le migrant célibataire, principalement de sexe masculin et relativement jeune (69\% ont moins de 30 ans), constitue donc un groupe qui se fondra à une communauté composée principalement de couples. Il devient essentiel de procéder à une description détaillée de ce couple migrant. A cette fin, nous avons retenu deux caractéristiques 
principales: la durée du mariage ainsi que le nombre d'enfants du couple lors de son arrivée.

Dans le premier cas (tableau 5), 36\% des couples sont mariés depuis moins de 5 ans alors que les couples avec 10 ans et plus de mariage apparaissent dans une proportion similaire (40\%). Il semble donc que les couples mariés depuis de nombreuses années affichent une mobilité pour le moins surprenante. En conséquence, ces couples se déplacent vraisemblablement avec des enfants. Le tableau 6 nous permet de préciser davantage cet aspect.

TABLEAU 5

Durée du mariage du couple à l'arrivée, par aire d'observation

\begin{tabular}{|lrrcccc|}
\hline \multicolumn{7}{c|}{ Durée du mariage } \\
\hline $\begin{array}{l}\text { Aire } \\
\text { d'observation }\end{array}$ & $\begin{array}{r}0-4 \\
\text { ans }\end{array}$ & $\begin{array}{c}5-9 \\
\text { ans }\end{array}$ & $\begin{array}{c}10-14 \\
\text { ans }\end{array}$ & $\begin{array}{c}15 \text { et }+ \\
\text { ans }\end{array}$ & $\begin{array}{c}\text { Ind. } \\
\text { Ensem- } \\
\text { ble }\end{array}$ \\
\hline Beaumont & 13 & 10 & 7 & 8 & 0 & 38 \\
Chambly & 0 & 1 & 1 & 1 & 0 & 3 \\
Deschambault & 10 & 9 & 6 & 5 & 1 & 31 \\
Les Éboulements & 5 & 3 & 0 & 1 & 0 & 9 \\
Gaspé & 0 & 1 & 1 & 2 & 0 & 4 \\
Lotbinière & 3 & 3 & 3 & 5 & 0 & 14 \\
Rivière-Ouelle & 15 & 5 & 5 & 8 & 0 & 33 \\
St-Joseph Beauce & 9 & 3 & 5 & 4 & 0 & 21 \\
\hline Ensemble & & & & & & \\
$\quad$ nombre absolu & 55 & 35 & 28 & 34 & 1 & 153 \\
nombre relatif $(\%)$ & 36 & 23 & 18 & 22 & - & 100 \\
\hline
\end{tabular}

Comme le laissait prévoir le tableau 5, les couples migrants possèdent un nombre relativement élevé d'enfants lors de leur installation. En effet, ils comptent en moyenne près de 4 enfants à leur arrivée. Le tableau 6 nous permet de constater que la taille de la famille ne semble pas être véritablement un obstacle à leur déplacement.

Les situations qui poussent les familles à migrer sont des plus diverses. Le jeune couple sans enfant qui cherche à s'installer à demeure est bien présent dans le processus migratoire. De plus, l'opportunité d'assurer éventuellement des terres à leurs enfants peut inciter des couples âgés à s'installer également dans ces nouvelles communautés. 
Pour conclure le portrait du migrant, il semble évident que la venue de couples exerce un poids considérable dans l'avènement de ces nou-

TABLEAU 6

Nombre d'enfants du couple à l'arrivée, par aire d'observation

\begin{tabular}{|lrrrrrrrrrr|}
\hline & \multicolumn{11}{c|}{ Nombre d'enfants } & \\
\hline $\begin{array}{l}\text { Aire } \\
\text { d'observation }\end{array}$ & 0 & 1 & 2 & 3 & 4 & 5 & 6 et + & Ind. & $\begin{array}{c}\text { Ensem- } \\
\text { ble }\end{array}$ \\
\hline Beaumont & 10 & 2 & 2 & 3 & 6 & 6 & 9 & 0 & 38 \\
Chambly & 0 & 0 & 0 & 0 & 0 & 2 & 1 & 0 & 3 \\
Deschambault & 7 & 4 & 3 & 2 & 4 & 2 & 8 & 1 & 31 \\
Les Éboulements & 0 & 3 & 4 & 1 & 0 & 0 & 1 & 0 & 9 \\
Gaspé & 0 & 1 & 2 & 0 & 1 & 0 & 0 & 0 & 4 \\
Lotbinière & 1 & 1 & 1 & 5 & 2 & 3 & 1 & 0 & 14 \\
Rivière-Ouelle & 6 & 7 & 1 & 7 & 4 & 4 & 4 & 0 & 33 \\
St-Joseph de Beauce & 4 & 3 & 2 & 2 & 1 & 1 & 8 & 0 & 21 \\
\hline Ensemble & & & & & & & & & \\
$\quad$ nombre absolu & 28 & 21 & 15 & 20 & 18 & 18 & 32 & 1 & 153 \\
nombre relatif $(\%)$ & 18 & 14 & 10 & 13 & 12 & 12 & 21 & - & 100 \\
\hline
\end{tabular}

velles communautés. Bien plus, l'arrivée de couples âgés avec des enfants en âge de s'établir pourrait permettre le développement de réseaux de parenté, consolidant ainsi des noyaux de familles bien enracinées. Les migrants célibataires y verraient alors l'opportunité de s'intégrer à une communauté en formation.

\section{4 - RÉSEAU DE PARENTÉ ET PERMANENCE}

La problématique de notre étude consiste à évaluer le rôle de la parenté au moment de l'arrivée du migrant et dans le processus d'enracinement. Certes, d'autres éléments interviennent également dans le choix d'un lieu d'établissement. Nous avons perçu lors de cette étude l'existence de solidarités de provenance. A titre d'exemples, signalons que la côte de Beaupré fournit $40 \%$ des pionniers de St-Joseph. C'est le cas également à Deschambault où $20 \%$ d'entre eux arrivent de SainteFamille de l'île d'Orléans. Dans cette présente étude, nous porterons 
toute notre attention à la solidarité familiale comme stratégie de colonisation.

Notre première démarche visa à évaluer l'importance des solidarités familiales pour chacune des communautés choisies. Ainsi, fallaitil pour chacun des migrants identifier s'il avait des parents à l'arrivée. Nous avons traduit ce phénomène par l'expression «taux de parenté à l'arrivée» (nombre de migrants ayant un parent à l'arrivée/nombre total de migrants). Le processus d'enracinement du migrant s'est exprimé par le «taux de permanence» (nombre total de permanents/nombre total de migrants). Le tableau 7 nous précise pour chacune des communautés les résultats obtenus.

TABLEAU 7

Taux de parenté à l'arrivée et de permanence des migrants, par aire d'observation

\begin{tabular}{|lcc|}
\hline Aire d'observation & $\begin{array}{c}\text { Parenté à l'arrivée } \\
\%\end{array}$ & $\begin{array}{c}\text { Permanence } \\
\%\end{array}$ \\
\hline Beaumont & 38 & 71 \\
Chambly & 0 & 28 \\
Deschambault & 34 & 58 \\
Les Éboulements & 75 & 69 \\
Gaspé & 24 & 4 \\
Lotbinière & 27 & 56 \\
Rivière-Ouelle & 45 & 70 \\
St-Joseph & 42 & 61 \\
\hline
\end{tabular}

Les liens de parenté jouent différemment, selon les zones concernées, dans l'établissement et l'installation des migrants. Ainsi, à Chambly comme à la côte de Gaspé, le faible taux de parenté à l'arrivée a pu concourir à l'instabilité des premiers migrants. Dans les deux cas, ce peuplement s'est inscrit également dans une politique dirigée de prise de possession du territoire (militaire et pêche).

La situation est tout à fait différente dans les six autres communautés agricoles. En effet, $41 \%$ des migrants avaient alors des liens de

\footnotetext{
$4 \quad$ Dans certaines seigneuries (Deschambault et Lotbinière entre autres), le taux de parenté calculé peut souffrir de l'arbitraire des limites seigneuriales. En effet, des arrivants déclarés sans parenté dans leur seigneurie de résidence auront un frère ou un beau-frère installé quelques terres plus loin, mais dans la seigneurie voisine.
} 
parenté avec des résidants. Il apparaît donc que les liens de parenté constituent véritablement un facteur d'attraction. L'établissement des nouveaux arrivants s'en trouve sans doute facilité car l'existence de liens familiaux leur permet de s'intégrer à une communauté en formation. D'ailleurs, le succès relatif de ces aires de colonisation (taux de permanence global de 64\%) semble indiquer que les liens familiaux favorisent l'établissement des nouveaux arrivants.

Existe-t-il d'autres facteurs liés à la parenté qui puissent influencer un migrant à devenir permanent que le seul fait d'avoir un parent sur place? Certes, il est impossible de suivre tous les destins individuels avec précision et surtout de connaître chaque fois les raisons qui incitent à partir ou à s'installer. Cependant, nous tenterons de cerner par le biais des solidarités familiales des éléments de réponse au comportement d'enracinement du migrant.

Quel groupe de migrants est le plus susceptible de devenir permanent? Existe-t-il un taux d'enracinement différent selon que le migrant est célibataire ou marié, qu'il ait un parent ou non à l'arrivée, ou qu'il se crée ou non des liens de parenté dans sa communauté?

Globalement, $64 \%$ des migrants deviennent permanents dans ces communautés. L'état matrimonial du migrant à l'arrivée fait ressortir des comportements différents. En effet, les migrants célibataires constituent le groupe où le taux d'enracinement est le plus faible (56\%). Les conjointes de résidants demeurent un groupe ayant un fort taux de permanence $(78 \%)$. Ce dernier résultat n'a rien pour surprendre car les conjointes de résidants, par définition, rejoignent un époux déjà sur place, et qui, dans maints cas y possèdent déjà des parents. Quant aux gens mariés, ils sont permanents dans près de deux cas sur trois (64\%). Une analyse plus élaborée du tableau 8 nous permet d'apporter certaines précisions.

En effet, les résultats deviennent particulièrement révélateurs lorsque nous établissons des comparaisons dans le taux de permanence en distinguant les migrants avec parenté à l'arrivée et ceux n'en ayant pas. $70 \%$ des migrants ayant de la parenté à l'arrivée deviennent permanents tandis que cette proportion tombe à $60 \%$ pour les migrants n'ayant aucun parent à l'arrivée. C'est donc dire que le fait d'avoir un parent sur place incite plus à la permanence mais ne constitue pas nécessairement un gage de stabilité car malgré tout $30 \%$ d'entre eux vont quitter.

Il semble plutôt que ce soit les liens de parenté créés dans le milieu qui prédominent avant tout. Les migrants qui se créent des liens familiaux, qu'ils aient de la parenté ou pas à l'arrivée, semblent les groupes les plus permanents, avec respectivement des taux de $82 \%$ et $75 \%$. Quant aux groupes de migrants qui ne se créent aucun lien de parenté, ils affichent des taux de permanence particulièrement bas: $36 \%$ pour 
TABLEAU 8

Permanence de l'arrivant selon l'état matrimonial à l'arrivée et le type de parenté

\begin{tabular}{|c|c|c|c|c|c|c|c|c|c|c|c|c|}
\hline \multirow[b]{4}{*}{ TYPE DE PARENTÉ } & \multicolumn{12}{|c|}{ TAUX DE PERMANENCE (\%) } \\
\hline & \multicolumn{12}{|c|}{ État matrimonial à l'arrivée } \\
\hline & \multicolumn{3}{|c|}{ Mariés et veufs } & \multicolumn{3}{|c|}{ Célibataires } & \multicolumn{3}{|c|}{ Conjointes de résidents } & \multicolumn{3}{|c|}{ Ensemble } \\
\hline & n.a. & n.t. & $\%$ & n.a. & n.t. & $\%$ & n.a. & n.t. & $\%$ & n.a. & n.t. & $\%$ \\
\hline $\begin{array}{l}\text { I- Sans parenté à l'arrivée } \\
\text { a) et qui ne s'en crée pas sur } \\
\text { place }\end{array}$ & 29 & 75 & 39 & 8 & 31 & 26 & 2 & 3 & 67 & 39 & 109 & 36 \\
\hline $\begin{array}{l}\text { b) et qui s'en crée ou en attire } \\
\text { sur place }\end{array}$ & 82 & 113 & 73 & 34 & 44 & 77 & 16 & 20 & 80 & 132 & 177 & 75 \\
\hline Ensemble & 111 & 188 & 59 & 42 & 75 & 56 & 18 & 23 & 78 & 171 & 286 & 60 \\
\hline $\begin{array}{l}\text { II- Avec parenté à l'arrivée } \\
\text { a) et qui ne s'en crée pas sur } \\
\text { place }\end{array}$ & 18 & 40 & 45 & 6 & 14 & 43 & 17 & 25 & 68 & 41 & 79 & 52 \\
\hline $\begin{array}{l}\text { b) et qui s'en crée ou en attire } \\
\text { sur place }\end{array}$ & 66 & 78 & 85 & 13 & 20 & 65 & 19 & 21 & 90 & 98 & 119 & 82 \\
\hline Ensemble & 84 & 118 & 71 & 19 & 34 & 56 & 36 & 46 & 78 & 139 & 198 & 70 \\
\hline ENSEMBLE & 195 & 306 & 64 & 61 & 109 & 56 & 54 & 69 & 78 & 310 & 484 & 64 \\
\hline
\end{tabular}

n.a.: nombre absolu n.t.: nombre total 
ceux n'ayant aucun parent à l'arrivée et $52 \%$ pour ceux ayant déjà un parent sur place. En somme, il semble exister une relation très nette entre la création de liens familiaux sur place et la permanence des arrivants.

Un autre exemple s'avère particulièrement révélateur, celui des célibataires sans parent à l'arrivée. Ceux qui ne réussissent pas à s'intégrer à une famille par le biais d'un mariage ne deviennent permanents que dans un cas sur quatre (26\%). A l'opposé, ceux qui, bien que sans lien à l'arrivée, s'en créent après ou attirent des parents, s'installent à demeure trois fois sur quatre (77\%). C'est donc dire l'importance pour les célibataires de créer des liens avec des familles déjà bien établies s'ils veulent s'intégrer à la communauté. Puisque $90 \%$ des célibataires sont des hommes, le rôle des femmes devient essentiel. Ce sont elles qui, dans bien des cas, permettent au mari de s'intégrer dans un réseau familial et qui par le fait même facilitent leur installation permanente.

Cette possibilité pour les célibataires de s'intégrer à un réseau met en lumière l'existence de noyaux stables formés par des familles anciennes comptant généralement plusieurs enfants, souvent assez âgés pour s'établir. Dans chacune des seigneuries agricoles, on retrouve de ces familles pionnières qui vont accaparer le terroir tout en permettant à des célibataires de s'intégrer.

Les solidarités de parenté ont donc un rôle de premier plan dans le processus d'intégration. Toutefois, il semble que les liens familiaux créés sur place ont nettement plus de poids que les liens antérieurs à la migration. Si elle vise la stabilité et l'enracinement, la stratégie de tout pionnier doit inclure la recherche de liens, principalement de liens familiaux, et sans cette dimension toute tentative d'occupation du sol risque de s'avérer fort vaine.

\section{CONCLUSION}

Dans une problématique visant à mieux connaître les pratiques sociales régissant la colonisation, nous avons cherché à démontrer le role dynamique de la parenté dans la formation d'une nouvelle communauté. Ce rôle apparaît en deux temps spécifiques: lors de l'arrivée et dans le processus d'enracinement.

En premier lieu, la motivation de migrer et de s'installer dans une nouvelle seigneurie résulte souvent de la présence d'un parent déjà rendu sur place. Cette situation mérite d'être explorée davantage car nous pourrions ainsi en connaître beaucoup plus sur la force relative des niveaux de parenté dans le phénomène d'attraction de l'arrivant. De même, il faudrait suivre de façon plus attentive les groupes d'arrivants liés entre eux afin de bien cerner leurs stratégies d'occupation du sol 
dans les premières années d'installation. Nous pourrions ainsi vérifier la durabilité du dynamisme des liens familiaux.

Quant au processus d'enracinement, on a vu l'impact décisif de la création de liens à l'intérieur de la communauté. Mais une analyse en profondeur reste à faire concernant l'importance des solidarités familiales. Quel est leur poids dans le choix des conjoints des migrants célibataires ou des enfants d'arrivants? Jusqu'à quel point influencentelles la sélection des parrains, marraines et tuteurs ou les conditions d'un éventuel remariage? Peuvent-elles jouer sur les modalités de transmission du patrimoine familial? Voilà autant de questions dont les réponses nous permettraient de mieux cerner les stratégies de reproduction de la famille comme celles de la société rurale dans son ensemble. 